\title{
Effect of polypharmacy, potentially inappropriate medications and anticholinergic burden on clinical outcomes: a retrospective cohort study
}

\author{
Wan-Hsuan Lu MS, Yu-Wen Wen PhD, Liang-Kung Chen MD PhD, Fei-Yuan Hsiao PhD
}

See also review, www.cmaj.ca/lookup/doi/10.1503/cmaj.131873

Competing interests: None declared.

This article has been peer reviewed.

Correspondence to: Fei-Yuan Hsiao, fyshsiao @ntu.edu.tw

CMAJ 2015. DOI:10.1503 /cmaj.141219

\begin{abstract}
Background: Polypharmacy, potentially inappropriate medications and anticholinergic burden (as assessed by the anticholinergic risk scale) are commonly used as quality indicators of pharmacotherapy in older adults. However, their role in clinical practice is undefined. We sought to investigate longitudinal changes in these indicators and their effects on clinical outcomes.
\end{abstract}

Methods: We used Taiwan's Longitudinal Health Insurance Database to retrieve quarterly information about drug use for people aged 65 years and older over a 10-year period. We analyzed the association between indicators and all-cause admission to hospital, fracture-specific admission to hospital and death using generalized estimating equations.

Results: The study cohort comprised 59042 older adults (65-74 yr: 39358 [66.7\%], $75-84$ yr: 16903 [28.6\%], and $\geq 85$ yr: 2781 $[4.7 \%]$ ). The mean changes in polypharmacy over the course of the study were greatest among patients aged 65-74 years (absolute difference $+2.14,95 \%$ confidence interval $[\mathrm{Cl}]$ 2.10-2.19), then among those aged 75-84 yr
$(+1.79,95 \% \mathrm{Cl} 1.70-1.88)$, and finally those aged 85 years and older $(+0.71,95 \% \mathrm{Cl} 0.36-$ 1.05). The number of potentially inappropriate medications increased among patients aged $65-74$ years $(+0.16[0.15-0.18])$ and $75-84$ years $(+0.09[0.06-0.08])$, but decreased in those aged 85 years and older $(-0.15[-0.26$ to -0.04$]$ ). Polypharmacy, potentially inappropriate medications and anticholinergic risk scale were each associated with an increased risk of admission to hospital, but not with death. In addition, both polypharmacy (5-9 drugs: odds ratio [OR] 1.18, 95\% Cl 1.12-1.24; $\geq 10$ drugs: OR $1.54,95 \% \mathrm{Cl} 1.42-1.66)$ and anticholinergic burden (score 1-2: 1.39, 95\% Cl 1.31-1.48; $\geq 3: 1.53$, 95\% Cl 1.41-1.66) showed dose-response relations with fracture-specific admission to hospital.

Interpretation: The total number of drugs taken (polypharmacy), number of potentially inappropriate medications and anticholinergic risk changed during follow-up and varied across age groups in this cohort of older adult patients. These indicators showed dose-response relations with admission to hospital, but not with death.
$\mathrm{P}$ opulation aging is a global phenomenon that poses various health care challenges. With the expected increase in multimorbidity and associated high consumption of drugs among older adults, understanding the quality of drug use for this population is essential. Although several indicators have been developed to evaluate the quality of pharmacotherapy in older adults, such as polypharmacy, ${ }^{1}$ potentially inappropriate medications ${ }^{2,3}$ and anticholinergic burden, ${ }^{4}$ several questions remain as to their utility in clinical practice.

Empirical data are scarce regarding changes in drug use with the increasing burden of multimorbidities in a single patient. Most studies have examined these quality indicators in a cross-sectional manner that provides one-time descriptive data ${ }^{1,5-8}$ or in a repeated crosssectional manner that provides descriptive data over time. ${ }^{9,10}$ In a few prospective cohort studies an attempt was made to overcome the limitations of cross-sectional studies, but they were limited by small sample sizes or short followup periods. ${ }^{11,12}$

Most of the previous studies have grouped people aged 65 years and older into a single age group. Empirical evidence regarding the potential variations in clinical conditions and treatments, as well as their effects on drug use among older adults at different ages, is scarce, and differences in drug use between older men and women have not been well investigated. 
The available studies have shown inconclusive findings in terms of the association between polypharmacy, potentially inappropriate medications or anticholinergic burden and possible adverse outcomes. ${ }^{13-15}$ Some studies have shown that polypharmacy or potentially inappropriate medications are positively associated with admission to hospital or death, ${ }^{13}$ while others have not. ${ }^{15}$ Moreover, little is known regarding the ability to predict negative outcomes among polypharmacy, potentially inappropriate medications or anticholinergic burden, because previous studies have focused on single indicators alone. ${ }^{13-15}$

Our aim was to examine changes in polypharmacy, potentially inappropriate medications and anticholinergic burden over time among people aged 65 years and older. In addition, we sought to determine the association between changes in these indicators and clinical outcomes, specifically all-cause admissions to hospital, fracturespecific admissions to hospital and death.

\section{Methods}

\section{Data source}

We performed a population-based retrospective cohort study using data from the National Health Insurance Research Database (NHIRD) - a nationwide claim-based database comprising anonymous enrollment information and health care usage data for more than $99 \%$ of the population of Taiwan. ${ }^{16}$ We used a subset of this database, the Longitudinal Health Insurance Database, which contains claims data for 1 million randomly selected beneficiaries from the NHIRD, to compose a 12-year (2000-2011) panel of claims for analysis.

Because the identification numbers for all of the entries in the NHRID were encrypted to protect the privacy of the individual patients, this study was exempt from a full review by the Institutional Review Board of the National Taiwan University Hospital, and informed consent was waived (National Taiwan University Hospital Research Ethics Committee No. 201401004W).

\section{Study cohort}

We identified people who were more than 65 years old and placed them into 1 of 3 groups (65-74, 75-84 and $\geq 85 \mathrm{yr}$ ) based on their age at the time of enrollment (Jan. 1, 2001). We excluded people with diagnoses within 1 year before the date of recruitment of any of 22 chronic diseases requiring long-term medical care and support, such as cancer, rheumatoid arthritis or systemic lupus erythematosus under Taiwan's National Health Insurance system. We made these exclusions owing to patients' risk of death in the short term (Appendix 1, available at www.cmaj.ca
/lookup/suppl/doi:10.1503/cmaj.141219/-/DC1). The first year after recruitment served as the baseline information-gathering period. The follow-up period for each participant started on Jan. 1, 2002, and ended on the date of death or on Dec. 31, 2011, whichever came first.

\section{Outcome measures}

During the 10-year follow-up period, we retrieved data on drug use quarterly (Q1-Q40) from the outpatient clinics in the Longitudinal Health Insurance Database. These data included prescribing dates, number of days' worth of drug supply and daily doses. We selected only those drugs with more than a 28-day supply to determine polypharmacy, potentially inappropriate medications or anticholinergic burden (as measured using the anticholinergic risk scale). All of the drugs were coded according to the World Health Organization (WHO) Anatomic Therapeutic Chemical classification system. ${ }^{17}$

We defined polypharmacy and excessive polypharmacy as the concomitant use of 5 or more and 10 or more drugs, respectively, based on previous studies. ${ }^{7,18}$ We defined potentially inappropriate medications using the 2012 update to the American Geriatrics Society's Beers Criteria. ${ }^{2,3}$ These criteria include 53 medications (classes) divided into 3 categories: medications to avoid in older adults, medications to avoid in older adults with certain diseases and syndromes, and medications to be used with caution in older adults. ${ }^{3} \mathrm{We}$ focused on the 34 medications to avoid in older adults. We determined anticholinergic burden using the anticholinergic risk scale, ${ }^{19}$ which ranks the 500 most frequently prescribed drugs with anticholinergic potential on a scale of $0-3(0, \mathrm{lim}-$ ited or none; 1 , moderate; 2 , strong; 3 , very strong potential) based on the dissociation constant for the muscarinic receptor and rates of adverse effects. A patient's score was the sum of the rankings for all prescribed medications. ${ }^{19}$

Our outcomes of interest were death, allcause admissions to hospital and fracturespecific admissions to hospital (International Classification of Diseases, 9th revision, clinical modification diagnostic codes 800-829).

\section{Other covariables}

In addition to the age and sex of participants, we retrieved health-related information (such as the number of outpatient visits) and determined the Charlson comorbidity index 1 year before the date of entry to the cohort to serve as proxies of participants' health statuses.

\section{Statistical analysis}

We used $\chi^{2}$ tests to compare categorical variables and $t$ tests or analyses of variance 
(ANOVA) to compare continuous variables. We used paired $t$ tests to examine differences in polypharmacy, potentially inappropriate medications and anticholinergic risk scale scores (mean and $95 \%$ confidence intervals $[\mathrm{CI}]$ ) between the end of follow-up and the beginning.

Because the mean of differences in polypharmacy, potentially inappropriate medications and anticholinergic burden were not normally distributed, we used the Kruskal-Wallis and Wilcoxon rank-sum tests to compare differences across age groups and sex.

We used the Chow test ${ }^{17}$ to examine differences in the time-varying use of polypharmacy, potentially inappropriate medications or anticholinergic risk by age and sex.
We used generalized estimating equation (GEE) models with an autoregressive correlation structure to examine the association between polypharmacy, potentially inappropriate medications or anticholinergic risk and clinical outcomes (i.e., death) that accounted for the intraclass correlation between repeated measurements for the same participants. We further adjusted these models for age, sex and time-varying comorbidities, but not for healthrelated resource use owing to concern regarding the high collinearity between it and drug exposure. We determined the likelihood of death or admission to hospital using a logit link with a binomial distribution (presented as the odds ratio [OR] with $95 \% \mathrm{CI}$ ). We performed all

Table 1: Baseline characteristics and health services use of participants

\begin{tabular}{|c|c|c|c|c|c|}
\hline \multirow{2}{*}{ Characteristic } & \multirow[b]{2}{*}{ Overall } & \multicolumn{3}{|c|}{ Age group, yr } & \multirow{2}{*}{$p$ value* } \\
\hline & & $65-74$ & $75-84$ & $\geq 85$ & \\
\hline Follow-up, person-years & 469514.25 & 338740.5 & 117737.5 & 13036.25 & \\
\hline Follow-up, person-years, mean (SD) & 7.95 (3.03) & $8.61 \quad(2.61)$ & $6.97 \quad(3.29)$ & $4.69 \quad(3.15)$ & \\
\hline $\begin{array}{l}\text { Charlson comorbidity index, } \\
\text { mean (SD) }\end{array}$ & 1.15 (1.31) & $1.10 \quad(1.28)$ & $1.27(1.36)$ & $1.20 \quad(1.32)$ & $<0.001$ \\
\hline \multicolumn{6}{|l|}{ Comorbidity, no. (\%) } \\
\hline Hypertension & $23279(39.4)$ & $15132(38.5)$ & $7094(42.0)$ & $1053(37.9)$ & $<0.001$ \\
\hline Diabetes & 10575 (17.9) & $7351(18.7)$ & $2884(17.1)$ & $340(12.2)$ & $<0.001$ \\
\hline Dementia & $1340 \quad(2.3)$ & 512 (1.3) & $628(3.7)$ & $200 \quad(7.2)$ & $<0.001$ \\
\hline Arthritis & $16636(28.2)$ & $10791(27.4)$ & $5052(29.9)$ & $793(28.5)$ & $<0.001$ \\
\hline Osteoporosis & $5693 \quad(9.6)$ & $3575 \quad(9.1)$ & $1783(10.5)$ & $335(12.0)$ & $<0.001$ \\
\hline \multicolumn{6}{|l|}{ Ambulatory care use, mean (SD) } \\
\hline No. of visits & $22.04(17.78)$ & $21.42(17.34)$ & $23.41(18.48)$ & $22.48(19.02)$ & $<0.001$ \\
\hline No. of visits (by specialty) & $3.35 \quad(1.90)$ & $3.36(1.91)$ & $3.39(1.91)$ & $3.07 \quad(1.74)$ & $<0.001$ \\
\hline No. of visits (by physician) & $5.38 \quad(3.79)$ & $5.33 \quad(3.77)$ & 5.55 (3.89) & $5.01 \quad(3.50)$ & $<0.001$ \\
\hline Drug use & & & & & \\
\hline No. of drugs, mean (SD) & $3.12(3.01)$ & $2.97 \quad(2.95)$ & $3.45(3.11)$ & $3.17 \quad(2.96)$ & $<0.001$ \\
\hline ARS score, mean (SD) & $0.27 \quad(0.81)$ & $0.25 \quad(0.78)$ & $0.30(0.86)$ & $0.28 \quad(0.80)$ & $<0.001$ \\
\hline$\geq 1$ anticholinergic agent, no. (\%) & $7461(12.6)$ & $4701(11.9)$ & $2367(14.0)$ & $393(14.1)$ & $<0.001$ \\
\hline
\end{tabular}


analyses using SAS, version 9.1.3 (SAS Institute Inc., Cary, NC).

\section{Results}

We identified 59042 older adults (39 358 [66.7\%] aged 65-74 yr, 16903 [28.6\%] aged 75-84 yr and 2781 [4.7\%] aged $\geq 85 \mathrm{yr}$ ) (Table 1). Women accounted for $48.3 \%$ of patients aged $65-74$, $48.6 \%$ of those aged $75-84$ years and $58.3 \%$ of those aged 85 years and older. Mean Charlson comorbidity index scores were 1.10, 1.27 and 1.20 for patients aged $65-74$ years, $75-84$ years and 85 years and older, respectively. Hypertension, cerebrovascular diseases and arthritis were frequently documented chronic conditions in all age groups. Dementia and osteoporosis were most prevalent (7.2\% and $12.0 \%$, respectively) among patients aged 85 years and older. Overall, patients had a mean of 22.0 outpatient visits per year. At baseline, patients aged 75-84 years used the most drugs (mean 3.45 [standard deviation (SD) 3.11]. The proportion of patients with polypharmacy was highest in the 75- to 84-year age group $(32.5 \%)$. Participants older than 75 years of age were more likely to receive potentially inappropriate medications ( $48.7 \%$ of patients aged $75-84 \mathrm{yr}$, $47.8 \%$ of patients aged $\geq 85 \mathrm{yr}$ ) and anticholinergic agents $(14.0 \%$ of patients aged $75-84 \mathrm{yr}$, $14.1 \%$ of patients $\geq 85 \mathrm{yr}$ ) than those aged $65-74$ years (Table 1; the proportions of patients in each exposure variable over the 10-year study period are presented in Appendix 2 (available at www .cmaj.ca/lookup/suppl/doi:10.1503/cmaj.141219 /-/DC1).
Over the 10-year follow-up, the change in the number of drugs used was greatest among patients aged 65-74 yr (+2.14, 95\% CI 2.10-2.19) (Table 2 ). The number of potentially inappropriate medications used increased among patients aged 65-74 $\mathrm{yr}(+0.16,95 \% \mathrm{CI} 0.15$ to 0.18$)$ and those aged $75-84 \mathrm{yr}(+0.09,95 \%$ CI 0.06 to 0.08$)$, but decreased among those aged 85 years and older $(-0.15,95 \%$ CI -0.26 to -0.04$)$. Anticholinergic risk increased in all age groups $(p<0.001)$, and the sizes of the changes in scores across groups were comparable $(p=0.4)$. We found a significantly greater increase in the number of drugs used, number of potentially inappropriate medications and anticholinergic risk scores in men than in women among those aged $65-74$ and $75-84$ years (Table 2, Figures 1 and 2). Changes over time in all 3 indicators of drug use varied by age and sex $(p<0.001)$, with the exception of anticholinergic risk in patients aged 65-74 v. 75-84 yr, $p=0.03$ ).

We found a dose-response relation between polypharmacy and all-cause admission to hospital (5-9 drugs: OR 1.34, 95\% CI 1.32-1.36; $\geq 10$ drugs: OR 1.98, 95\% CI 1.94-2.03) (Table 3). Use of potentially inappropriate medications was associated with a $27 \%$ increase in the risk of allcause admission to hospital (OR 1.27, 95\% CI 1.25-1.28). Anticholinergic risk scores of 1-2 and 3 or more were associated with a $46 \%$ and $32 \%$ increase, respectively, in risk of all-cause admission to hospital. Both polypharmacy (5-9 drugs: OR 1.18, 95\% CI 1.12-1.24; $\geq 10$ drugs: OR $1.54,95 \%$ CI 1.42-1.66) and anticholinergic risk scale scores (1-2: OR $1.39,95 \%$ CI $1.31-$ $1.48 ; \geq 3$ : OR $1.53,95 \%$ CI $1.41-1.66)$ showed

Table 2: Changes in drug use during 10-year follow-up, stratified by age

\begin{tabular}{|c|c|c|c|c|c|}
\hline Indicator & $\begin{array}{c}\text { Overall, } \\
\text { mean }(95 \% \mathrm{Cl}) \\
n=36628\end{array}$ & \multicolumn{3}{|c|}{ Age group, yr, mean $(95 \% \mathrm{Cl})$} & $p$ value* \\
\hline No. of drugs & 2.05 (2.01 to 2.09 ) & 2.14 (2.10 to 2.19$)$ & 1.79 (1.70 to 1.88$)$ & 0.71 (0.36 to 1.05$)$ & $<0.001$ \\
\hline Women & 1.88 (1.83 to 1.93$)$ & 1.97 (1.91 to 2.03$)$ & $1.64(1.52$ to 1.76$)$ & 0.61 (0.21 to 1.01$)$ & \\
\hline Men & 2.24 (2.18 to 2.30$) \dagger$ & $2.33(2.27$ to 2.40$) \dagger$ & $1.96(1.82$ to 2.09$) \dagger$ & 0.86 (0.23 to 1.48$)$ & \\
\hline Women & $0.10(0.08$ to 0.11$)$ & 0.11 (0.09 to 0.13$)$ & $0.06(0.02$ to 0.10$)$ & $-0.12(-0.25$ to 0.01$)$ & \\
\hline Men & $0.19(0.18$ to 0.21$) \dagger$ & $0.22(0.20$ to 0.24$) \dagger$ & 0.12 (0.08 to 0.17$) \ddagger$ & $-0.20(-0.39$ to 0.00$)$ & \\
\hline ARS score & $0.19(0.18$ to 0.21$)$ & 0.20 (0.18 to 0.21$)$ & $0.19(0.16$ to 0.22$)$ & $0.14 \quad(0.04$ to 0.23$)$ & 0.4 \\
\hline Women & $0.18(0.16$ to 0.19$)$ & $0.18(0.16$ to 0.20$)$ & $0.17(0.13$ to 0.20$)$ & 0.11 (0.01 to 0.21$)$ & \\
\hline
\end{tabular}


dose-response relations with fracture-specific admission to hospital. The use of potentially inappropriate medications was associated with a $55 \%$ increase in the risk of fracture-specific admission (OR 1.55, 95\% CI 1.48-1.62). No such association was found between the 3 indicators and death.

\section{Interpretation}

In this cohort of patients aged 65 years and older followed for 10 years, polypharmacy, potentially inappropriate medications and anticholinergic burden changed over time and varied across age groups and sex. We found good

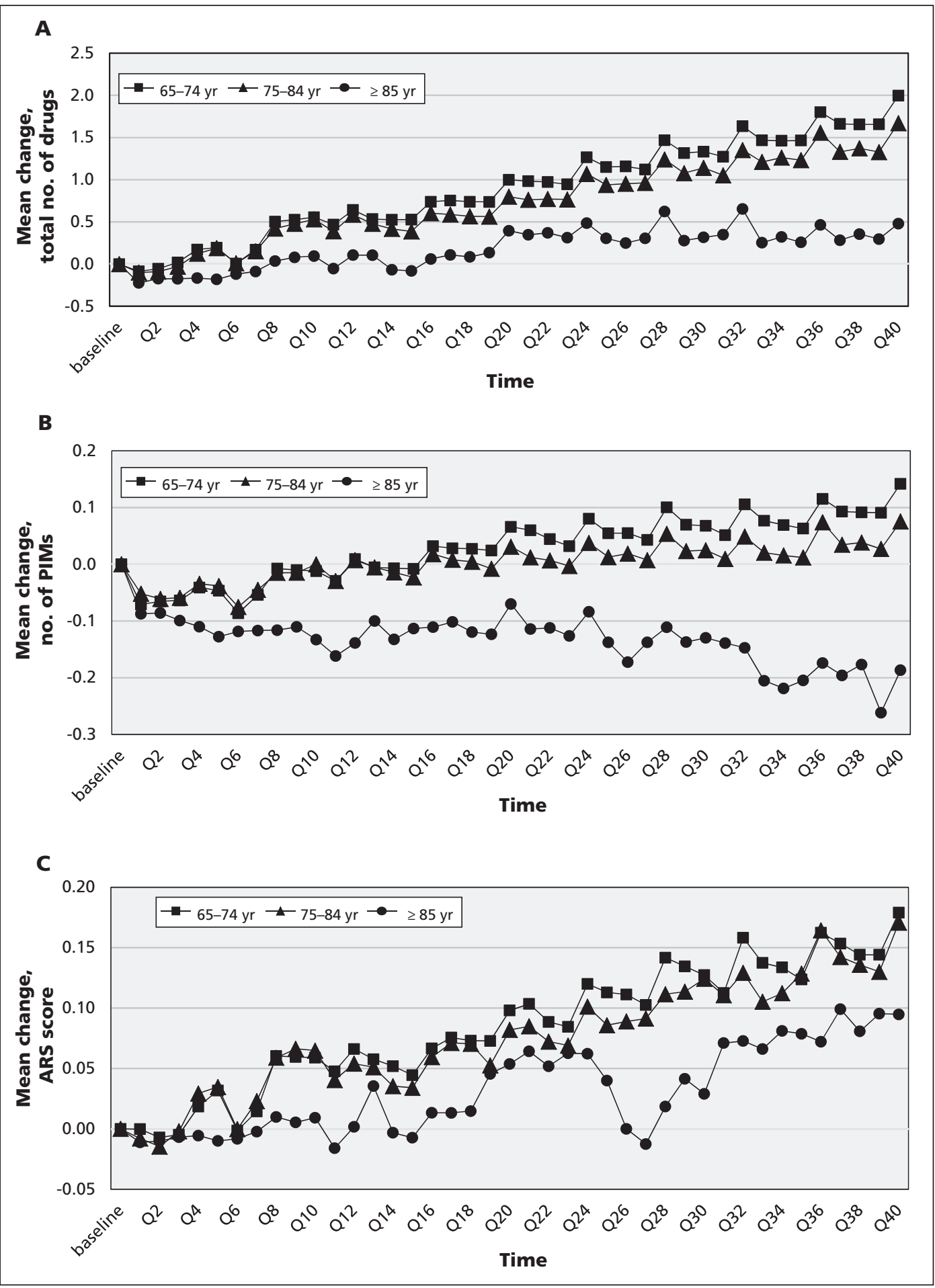

Figure 1: Mean changes in polypharmacy (A), use of PIMs (B) and anticholinergic burden as determined by ARS score (C) over a 10-year quarterly follow-up, stratified by age. ARS = anticholinergic risk scale, PIM = potentially inappropriate medication. 
associations between these quality indicators of drug use and both all-cause and fracture-specific admissions to hospital.

By combining the results for our 3 age groups, we have presented the consumption of drugs 30 years after an initial age of 65 years $(65-95$ years old). Previous studies have addressed similar concepts, but have been limited by small sample sizes and short follow-up periods. ${ }^{11,20}$ In a retrospective cohort study involving 1544 patients, Veehof and colleagues reported an increase in the prevalence of polypharmacy (26.4\% to $41.1 \%$; number of drugs used, mean [SD]: 1.3 [1.6] to 1.7 $[1.8]),{ }^{20}$ whereas Lapi and colleagues' prospective cohort study involving 568 patients showed an increase in drug use (mean [SD]: 2.0 [0.1] to 2.9 $[0.1]$ ), but a decrease in use of potentially inappropriate medications (mean [SD]: 0.09 [0.01] to 0.05 [0.01]) after 4 years of follow-up. ${ }^{11}$ Thus, we add to the current literature owing to our use of a larger sample and extended follow-up period.

In addition, we documented changes over time in anticholinergic burden in community-dwelling older adults. Our results from patients aged 85 years and older suggest that physicians may be more likely to withhold treatments in this age group owing to limited life expectancy or high vulnerability to treatment-related adverse events.

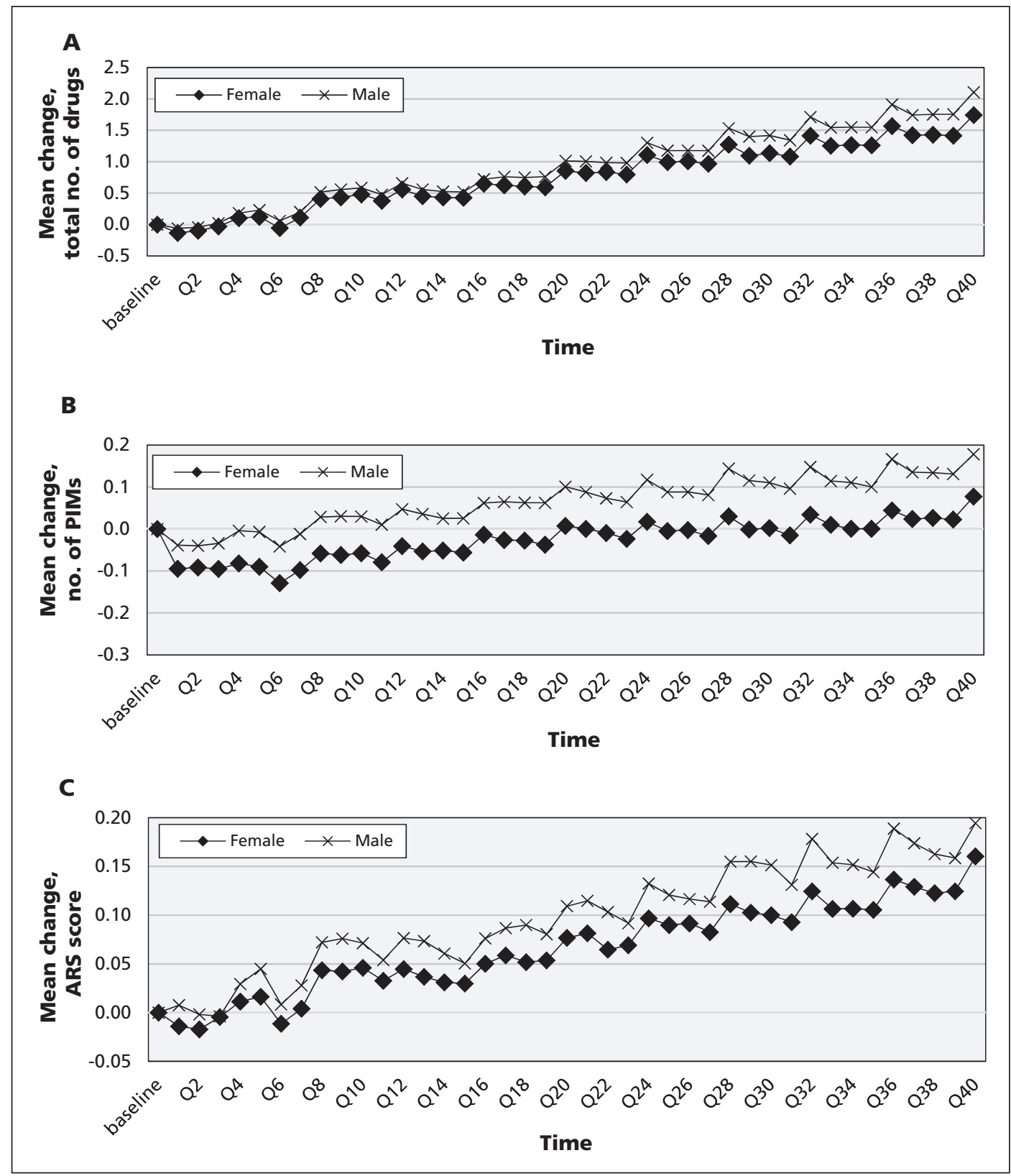

Figure 2: Mean changes in polypharmacy (A), use of PIMs (B) and anticholinergic burden as determined by ARS score (C) over a 10-year quarterly follow-up, stratified by sex. ARS = anticholinergic risk scale, PIM = potentially inappropriate medication. 
We found dose-response relations between polypharmacy or potentially inappropriate medications and all-cause admission to hospital. However, Pozzi and colleagues reported that potentially inappropriate medications (hazard ratio [HR] 1.03, 95\% CI 1.00-1.06), but not polypharmacy (HR 0.77, 95\% CI 0.58-1.01), were associated with admission to hospital. ${ }^{15}$ This discrepancy may result from the measurement of drug exposure. We measured drug use quarterly during follow-up and included changes in drug use in our models as time-varying variables, whereas Pozzi and colleagues used 2-wave cross-sectional measurements of drug use at baseline to predict subsequent admissions to hospital. ${ }^{15}$

Our results regarding dose-response relations between polypharmacy or potentially inappropriate medications and fracture-specific admissions to hospital are in accordance with previous studies. ${ }^{21,22}$ Most of those studies focused on the link between anticholinergic burden and cognitive function; ${ }^{12,23}$ thus, our study expands the potential use of anticholinergic risk scale by showing that a higher score was associated with a higher risk of admission to hospital. Some overlapping may have occurred in potentially inappropriate medications or anticholinergic burden. Koyama and colleagues reported that among community-dwelling women aged 75 years and older, the most frequently reported potentially inappropriate medications were anticholinergic agents (15.2\%). In addition,
Lee and colleagues reported that patients with $6-10$ or 11 or more prescribed medications were significantly more likely to receive prescriptions for anticholinergic agents (OR 3.41, 95\% CI 2.235.22 and OR 4.69, 95\% CI 2.99-7.34, respectively). ${ }^{23}$ Therefore, because we found a similar ability to predict fracture-specific admissions to hospital with use of each of 10 or more drugs, potentially inappropriate medications and anticholinergic burden, polypharmacy may serve as a quick quality indicator of drug use in older adults.

The associations of these quality indicators with risk of death remain unclear. Espino and colleagues reported an increased risk of death associated with polypharmacy (HR 1.27, 95\% CI 1.04-1.56) in a cohort study with an 8 -year follow-up period. ${ }^{13}$ However, Pozzi and colleagues (HR 1.20, 95\% CI $0.89-1.60)^{15}$ and Jyrkkä and colleagues (polypharmacy HR $0.77,95 \%$ CI $0.58-1.01$; excessive polypharmacy HR 1.28 95\% CI 0.86-1.91) ${ }^{1}$ reported no such association. Furthermore, Pozzi and colleagues suggested that potentially inappropriate medications were not associated with an increased risk of death (HR 1.00, 95\% CI 0.96-1.06). ${ }^{15} \mathrm{We}$ found that polypharmacy ( $\geq 10$ drugs), potentially inappropriate medications and elevated anticholinergic burden were associated with a reduced risk of death. One explanation for this phenomenon is the high number of patients who spend their final days in hospitals in Taiwan, and the daily variation in their drug use owing to urgent conditions. Because

\begin{tabular}{|c|c|c|c|c|c|c|c|c|c|}
\hline \multirow[b]{2}{*}{ Indicator } & \multicolumn{3}{|c|}{$\begin{array}{l}\text { All-cause admission to hospital } \\
\qquad n=162264\end{array}$} & \multicolumn{3}{|c|}{$\begin{array}{l}\text { Fracture-specific admission to hospital } \\
\qquad n=8220\end{array}$} & \multicolumn{3}{|c|}{$\begin{array}{c}\text { Death } \\
n=24740\end{array}$} \\
\hline & $\begin{array}{l}\text { No. of } \\
\text { patients }\end{array}$ & $\begin{array}{c}\mathrm{OR} \\
(95 \% \mathrm{Cl})\end{array}$ & $p$ value & $\begin{array}{l}\text { No. of } \\
\text { patients }\end{array}$ & $\begin{array}{c}\text { OR } \\
(95 \% \mathrm{Cl})\end{array}$ & $p$ value & $\begin{array}{l}\text { No. of } \\
\text { patients }\end{array}$ & $\begin{array}{c}\mathrm{OR} \\
(95 \% \mathrm{Cl})\end{array}$ & $p$ value \\
\hline \multicolumn{10}{|c|}{$\begin{array}{l}\text { Polypharmacy, } \\
\text { no. of drugs }\end{array}$} \\
\hline $1-4$ & 42256 & 1.00 & & 2877 & 1.00 & & 4463 & 1.00 & \\
\hline $5-9$ & 64902 & $\begin{array}{c}1.34 \\
(1.32-1.36)\end{array}$ & $<0.001$ & 3306 & $\begin{array}{c}1.18 \\
(1.12-1.24)\end{array}$ & $<0.001$ & 5317 & $\begin{array}{c}0.97 \\
(0.93-1.01)\end{array}$ & 0.1 \\
\hline$\geq 10$ & 27612 & $\begin{array}{c}1.98 \\
(1.94-2.03)\end{array}$ & $<0.001$ & 1056 & $\begin{array}{c}1.54 \\
(1.42-1.66)\end{array}$ & $<0.001$ & 1131 & $\begin{array}{c}0.59 \\
(0.56-0.64)\end{array}$ & $<0.001$ \\
\hline \multicolumn{10}{|c|}{ Use of PIMs } \\
\hline No & 71946 & 1.00 & & 3910 & 1.00 & & 17987 & 1.00 & \\
\hline Yes & 90318 & $\begin{array}{c}1.27 \\
(1.25-1.28)\end{array}$ & $<0.001$ & 4910 & $\begin{array}{c}1.55 \\
(1.48-1.62)\end{array}$ & $<0.001$ & 6753 & $\begin{array}{c}0.39 \\
(0.38-0.40)\end{array}$ & $<0.001$ \\
\hline \multicolumn{10}{|c|}{ ARS score } \\
\hline 0 & 122302 & 1.00 & & 6904 & 1.00 & & 21603 & 1.00 & \\
\hline $1-2$ & 27123 & $\begin{array}{c}1.46 \\
(1.43-1.49)\end{array}$ & $<0.001$ & 1250 & $\begin{array}{c}1.39 \\
(1.31-1.48)\end{array}$ & $<0.001$ & 2350 & $\begin{array}{c}0.75 \\
(0.72-0.79)\end{array}$ & $<0.001$ \\
\hline$\geq 3$ & 12839 & $\begin{array}{c}1.32 \\
(1.28-1.35)\end{array}$ & $<0.001$ & 666 & $\begin{array}{c}1.53 \\
(1.41-1.66)\end{array}$ & $<0.001$ & 787 & $\begin{array}{c}0.49 \\
(0.45-0.53)\end{array}$ & $<0.001$ \\
\hline
\end{tabular}


we only identified drugs with a supply period of more than 28 days to measure our 3 quality indicators, we were unable to capture end-of-life drug use. Our results showed that patients using 10 or more medications, patients using potentially inappropriate medications and patients with high anticholinergic burden were at a lower risk of death.

\section{Limitations}

Our use of numbers and amounts of medicine prescribed to define exposure presumes that patients always take the medicines they have been given. In addition, because we relied on claims data, we were unable to include drugs that are not covered by National Health Insurance, such as over-thecounter medications, herbal supplements and alternative remedies. Furthermore, we were not able to include variables that are not routinely captured in the claims database, such as tobacco or alcohol consumption. Finally, because the NHIRD only recorded up to 5 diagnoses per patient visit, ${ }^{18}$ we may have underestimated the prevalence of comorbidities in our cohort.

\section{Conclusion}

Prescribing patterns among this group of older adult patients from Taiwan changed during the follow-up period and varied across age groups and sex. We found dose-response relations between all 3 indicators of quality of drug use (polypharmacy, use of potentially inappropriate medications and anticholinergic burden) and admission to hospital (all-cause and fracture-specific), but not death. These findings are relevant to both clinicians and policy-makers, because a re-evaluation of the role of polypharmacy, potentially inappropriate medications and anticholinergic burden in older adults may be warranted.

\section{References}

1. Jyrkkä J, Enlund H, Korhonen MJ, et al. Patterns of drug use and factors associated with polypharmacy and excessive polypharmacy in elderly persons: results of the Kuopio 75+ study: a cross-sectional analysis. Drugs Aging 2009;26:493-503.

2. Fick DM, Cooper JW, Wade WE, et al. Updating the Beers criteria for potentially inappropriate medication use in older adults: results of a US consensus panel of experts. Arch Intern Med 2003;163:2716-24.

3. American Geriatrics Society 2012 Beers Criteria Update Expert Panel. American Geriatrics Society updated Beers Criteria for potentially inappropriate medication use in older adults. $J \mathrm{Am}$ Geriatr Soc 2012;60:616-31.

4. Rudd KM, Raehl CL, Bond CA, et al. Methods for assessing drug-related anticholinergic activity. Pharmacotherapy 2005;25:1592-601.

5. Fialová D, Topinkova E, Gambassi G, et al. Potentially inappropriate medication use among elderly home care patients in Europe. JAMA 2005;293:1348-58.

6. Hajjar ER, Cafiero AC, Hanlon JT. Polypharmacy in elderly patients. Am J Geriatr Pharmacother 2007;5:345-51.

7. Steinman MA, Landefeld CS, Rosenthal GE, et al. Polypharmacy and prescribing quality in older people. J Am Geriatr Soc 2006; 54:1516-23.

8. Zhan C, Sangl J, Bierman AS, et al. Potentially inappropriate medication use in the community-dwelling elderly: findings from the 1996 Medical Expenditure Panel Survey. JAMA 2001;286:2823-9.

9. Franchi C, Tettamanti M, Pasina L, et al. Changes in drug prescrib- ing to Italian community-dwelling elderly people: the EPIFARMElderly Project 2000-2010. Eur J Clin Pharmacol 2014;70:437-43.

10. Sumukadas D, McMurdo ME, Mangoni AA, et al. Tempora trends in anticholinergic medication prescription in older people: repeated cross-sectional analysis of population prescribing data. Age Ageing 2014;43:515-21.

11. Lapi F, Pozzi C, Mazzaglia G, et al. Epidemiology of suboptimal prescribing in older, community dwellers: a two-wave, populationbased survey in Dicomano, Italy. Drugs Aging 2009;26:1029-38.

12. Koyama A, Steinman M, Ensrud K, et al. Ten-year trajectory of potentially inappropriate medications in very old women: importance of cognitive status. J Am Geriatr Soc 2013;61:258-63.

13. Espino DV, Bazaldua OV, Palmer RF, et al. Suboptimal medication use and mortality in an older adult community-based cohort: results from the Hispanic EPESE Study. J Gerontol A Biol Sci Med Sci 2006;61:170-5.

14. Richardson K, Ananou A, Lafortune L, et al. Variation over time in the association between polypharmacy and mortality in the older population. Drugs Aging 2011;28:547-60.

15. Pozzi C, Lapi F, Mazzaglia G, et al. Is suboptimal prescribing a risk factor for poor health outcomes in community-dwelling elders? The ICARe Dicomano study. Pharmacoepidemiol Drug Saf 2010;19:954-60.

16. Hsiao FY, Yang CL, Huang YT, et al. Using Taiwan's national health insurance research databases for pharmacoepidemiology research. J Food Drug Anal 2007;15:99.

17. Chow GC. Tests of equality between sets of coefficients in 2 linear regressions. Econometrica 1960;28:591-605.

18. Haider SI, Johnell K, Weitoft GR, et al. The influence of educational level on polypharmacy and inappropriate drug use: a register-based study of more than 600000 older people. J Am Geriatr Soc 2009;57:62-9.

19. Rudolph JL, Salow MJ, Angelini MC, et al. The anticholinergic risk scale and anticholinergic adverse effects in older persons. Arch Intern Med 2008;168:508-13.

20. Veehof L, Stewart R, Haaijer-Ruskamp F, et al. The development of polypharmacy. A longitudinal study. Fam Pract 2000;17:261-7.

21. Baranzini F, Poloni N, Diurni M, et al. Polypharmacy and psychotropic drugs as risk factors for falls in long-term care setting for elderly patients in Lombardy [article in Italian]. Recenti Prog Med 2009;100:9-16.

22. Lai SW, Liao KF, Liao CC, et al. Polypharmacy correlates with increased risk for hip fracture in the elderly: a population-based study. Medicine 2010;89:295-9.

23. Lee EK, Lee YJ. Prescription patterns of anticholinergic agents and their associated factors in Korean elderly patients with dementia. Int J Clin Pharm 2013;35:711-8.

Affiliations: Graduate Institute of Clinical Pharmacy (Lu, Hsiao), College of Medicine, National Taiwan University, Taipei, Taiwan; Clinical Informatics and Medical Statistics Research Center (Wen), Chang Gung University, Taoyuan, Taiwan; Aging and Health Research Center (Chen), National Yang Ming University, Taipei, Taiwan; Center for Geriatrics and Gerontology (Chen), Taipei Veterans General Hospital, Taipei, Taiwan; School of Pharmacy, College of Medicine (Hsiao), National Taiwan University, Taipei, Taiwan; Department of Pharmacy (Hsiao), National Taiwan University Hospital, Taipei, Taiwan

Contributors: Wan-Hsuan Lu, Yu-Wen Wen, Liang-Kung Chen and Fei-Yuan Hsiao helped design the study. WanHsuan Lu, Yu-Wen Wen and Fei-Yuan Hsiao acquired and analyzed the data. Wan-Hsuan Lu, Yu-Wen Wen, LiangKung Chen and Fei-Yuan Hsiao wrote and revised the paper. All of the authors approved the final version and agreed to act as guarantors of the work.

Data sharing: Data are available on written request to the corresponding author.

Acknowledgements: The authors thank the National Health Insurance Administration (NHIA) and National Health Research Institutes (NHRI) for making available the databases used in this study. The content of this article in no way represents any official position of the NHIA or NHRI. FeiYuan Hsiao received research assistantships from a research project (NSC101-3114-Y-002-003) sponsored by the National Science Council, Taiwan, and a research project (MOHW103-FDA-41100) sponsored by the Food and Drug Administration (FDA) in Taiwan. The author had full access to all of the data in the study and takes responsibility for the integrity of the data and the accuracy of their analysis. 